\title{
On a hybrid algorithm for a family of total quasi- $\varphi$-asymptotically nonexpansive mappings in Banach spaces
}

Ping Zuo ${ }^{1}$, Shih-sen Chang ${ }^{2^{*}}$ and Min Liu ${ }^{1 *}$

\footnotetext{
* Correspondence: changss@yahoo. cn; liuminybsc@yahoo.com.cn

${ }^{1}$ Department of Mathematics, Yibin university, Yibin, Sichuan 644007, China

${ }^{2}$ Department of Mathematics, College of Statistics and

Mathematics, Yunnan University of Finance and Economics, Kunming, Yunnan 650221, China

Full list of author information is available at the end of the article
}

\begin{abstract}
The purpose of this article is to introduce the concept of total quasi- $\varphi$ asymptotically nonexpansive mapping which contains many kinds of mappings as its special cases and we prove a strong convergence theorem by using a hybrid method for finding a common element of the set of solutions for a generalized mixed equilibrium problems, the set of fixed points of a family of total quasi- $\varphi$ asymptotically nonexpansive mappings in uniformly smooth and strictly convex Banach space with the Kadec-Klee property. The results presented in the article improve and extend some recent results.

MR (2000) Subject Classification: 47H09; 47H05.
\end{abstract}

Keywords: total quasi- $\varphi$-asymptotically nonexpansive mapping, quasi- $\varphi$-asymptotically nonexpansive mapping, generalized mixed equilibrium problem, hybrid method.

\section{Introduction}

Let $E$ be a Banach space and $C$ be a closed convex subsets of $E$. Let $F$ be an equilibrium bifunction from $C \times C$ into $R, \psi: C \rightarrow R$ be a real-valued function and $A: C \rightarrow$ $E^{*}$ be a nonlinear mapping. The "so-called" generalized mixed equilibrium problem is to find $z \in C$ such that

$$
F(z, y)+\langle A z, y-z\rangle+\psi(y)-\psi(z) \geq 0, \forall y \in C
$$

The set of solutions of (1.1) is denoted by GMEP, i.e.,

$$
G M E P=\{z \in C: F(z, y)+\langle A z, y-z\rangle+\psi(y)-\psi(z) \geq 0, \forall y \in C\} .
$$

Special examples:

(I) If $A=0$, then the problem (1.1) is equivalent to find $z \in C$ such that

$$
F(z, y)+\psi(y)-\psi(z) \geq 0, \forall y \in C .
$$

which is called the mixed equilibrium problem, see [1]. The set of solutions of (1.2) is denoted by MEP.

(II) If $F=0$, then the problem (1.1) is equivalent to find $z \in C$ such that

$$
\langle A z, y-z\rangle+\psi(y)-\psi(z) \geq 0, \forall y \in C .
$$

\section{Springer}

(C) 2012 Zuo et al; licensee Springer. This is an Open Access article distributed under the terms of the Creative Commons Attribution License (http://creativecommons.org/licenses/by/2.0), which permits unrestricted use, distribution, and reproduction in any medium, provided the original work is properly cited. 
which is called the mixed variational inequality of Browder type. The set of solutions of (1.3) is denoted by $V I(C, A, \psi)$.

(III) If $\psi=0$, then the problem (1.1) is equivalent to find $z \in C$ such that

$$
F(z, y)+\langle A z, y-z\rangle \geq 0, \forall y \in C .
$$

which is called the generalized equilibrium problem, see [2]. The set of solutions of (1.4) is denoted by EP.

(IV) If $A=0, \psi=0$, then the problem (1.1) is equivalent to find $z \in C$ such that

$$
F(z, y) \geq 0, \forall y \in C .
$$

which is called the equilibrium problem. The set of solutions of (1.5) is denoted by $\mathrm{EP}(\mathrm{F})$.

These show that the problem (1.1) is very general in the sense that numerous problems in physics, optimization, and economics reduce to finding a solution of (1.1). Recently, some methods have been proposed for the generalized mixed equilibrium problem in Banach spaces (see, for example [1-7]).

Let $E$ be a smooth, strictly convex, and reflexive Banach spaces and $C$ be a nonempty closed convex subsets of $E$. Throughout this article, we denote by $\varphi$ the function defined by

$$
\phi(y, x)=\|y\|^{2}-2\langle y, J x\rangle+\|x\|^{2}, \forall x, y \in E,
$$

where $J: E \rightarrow 2^{E^{*}}$ is the normalized duality mapping.

Let $T: C \rightarrow C$ be a mapping and $F(T)$ be the set of fixed points of $T$.

Recall that a point $p \in C$ is said to be an asymptotic fixed point of $T$ if there exists $\left\{x_{n}\right\}$ in $C$ which converges weakly to $p$ and $\lim _{n \rightarrow \infty}|| x_{n}-T x_{n} \|=0$. We denote the set of all asymptotic fixed point of $T$ by $\tilde{F}(T)$. A point $p \in C$ is said to be a strong asymptotic fixed point of $T$ if there exists $\left\{x_{n}\right\}$ in $C$ such that $x_{n} \rightarrow p$ and $\lim _{n \rightarrow \infty}\left\|x_{n}-T x_{n}\right\|$ $=0$ We denote the set of all strongly asymptotic fixed point of $T$ by $\hat{F}(T)$.

A mapping $T: C \rightarrow C$ is said to be nonexpansive, if

$$
\|T x-T y\| \leq\|x-y\|, \forall x, y \in C .
$$

A mapping $T: C \rightarrow C$ is said to be relatively nonexpansive if $F(T) \neq \varnothing, F(T)=\tilde{F}(T)$, and

$$
\phi(u, T x) \leq \phi(u, x), \forall u \in F(T), x \in C
$$

A mapping $T: C \rightarrow C$ is said to be weak relatively nonexpansive if $F(T) \neq \varnothing$, $F(T)=\hat{F}(T)$, and

$$
\phi(u, T x) \leq \phi(u, x), \forall u \in F(T), x \in C
$$

A mapping $T: C \rightarrow C$ is said to be quasi- $\varphi$-nonexpansive, if $F(T) \neq \varnothing$ and

$$
\phi(u, T x) \leq \phi(u, x), \forall x \in C, \forall u \in F(T)
$$

A mapping $T: C \rightarrow C$ is said to be quasi- $\varphi$-asymptotically nonexpansive, if there exists some real sequence $\left\{k_{n}\right\}$ with $k_{n} \geq 1$ and $k_{n} \rightarrow 1$ and $F(T) \neq \varnothing$ such that 


$$
\phi\left(u, T^{n} x\right) \leq k_{n} \phi(u, x), \forall n \geq 1, x \in C, u \in F(T)
$$

A mapping $T: C \rightarrow C$ is said to be closed, if for any sequence $\left\{x_{n}\right\} \subset C$ with $x_{n} \rightarrow x$ and $T x_{n} \rightarrow y$, then $T x=y$.

Definition 1.1 (1) A mapping $T: C \rightarrow C$ is said to be total quasi- $\varphi$-asymptotically nonexpansive, if $F(T) \neq \varnothing$ and there exist nonnegative real sequences $\left\{v_{n}\right\},\left\{\mu_{n}\right\}$ with $v_{n} \rightarrow 0, \mu_{n} \rightarrow 0($ as $n \rightarrow \infty)$ and a strictly increasing continuous function $\zeta: \mathfrak{R}^{+} \rightarrow \mathfrak{R}^{+}$ with $\zeta(0)=0$ such that for all $x \in C, p \in F(T)$

$$
\phi\left(p, T^{n} x\right) \leq \phi(p, x)+v_{n} \zeta(\phi(p, x))+\mu_{n}, \forall n \geq 1 .
$$

(2) A family of mappings $\left\{T_{\lambda}\right\}_{\lambda \in \Lambda}: C \rightarrow C$ is said to be uniformly total quasi- $\varphi$ asymptotically nonexpansive, if $n_{\lambda \in \Lambda} F\left(T_{\lambda}\right) \neq \varnothing$ and there exist nonnegative real sequences $\left\{v_{n}\right\},\left\{\mu_{n}\right\}$ with $v_{n} \rightarrow 0, \mu_{n} \rightarrow 0$ (as $\left.n \rightarrow \infty\right)$ and a strictly increasing continuous function $\zeta: \mathfrak{R}^{+} \rightarrow \mathfrak{R}^{+}$with $\zeta(0)=0$ such that for all $x \in C, p \in \cap_{\lambda \in \Lambda} F\left(T_{\lambda}\right)$

$$
\phi\left(p, T_{i}^{n} x\right) \leq \phi(p, x)+v_{n} \zeta(\phi(p, x))+\mu_{n}, \forall n \geq 1 .
$$

Remark 1.1 From the definitions, it is easy to know that

(1) Each relatively nonexpansive mapping is closed;

(2) Taking $\zeta(t)=t, t \geq 0, v_{n}=\left(k_{n}-1\right)$ and $\mu_{n}=0$, then (1.7) can be rewritten as

$$
\phi\left(p, T^{n} x\right) \leq \phi(p, x)+v_{n} \zeta(\phi(p, x))+\mu_{n}, \forall n \geq 1, x \in C, p \in F(T) .
$$

This implies that each quasi- $\varphi$-asymptotically nonexpansive mapping must be a total quasi- $\varphi$-asymptotically nonexpansive mapping, but the converse is not true;

(3) The class of quasi- $\varphi$-asymptotically nonexpansive mappings contains properly the class of quasi- $\varphi$-nonexpansive mappings as a subclass, but the converse is not true;

(4) The class of quasi- $\varphi$-nonexpansive mappings contains properly the class of weak relatively nonexpansive mappings as a subclass, but the converse is not true;

(5) The class of weak relatively nonexpansive contains properly the class of relatively nonexpansive mappings as a subclass, but the converse is not true.

Iterative approximation of fixed points for relatively nonexpansive mappings, weak relatively nonexpansive mappings, quasi- $\varphi$ nonexpansive mappings, quasi- $\varphi$-asymptotically non-expansive mappings in the setting of Banach spaces has been studied extensively by many authors (see [5-13]).

Motivated by the above, the purpose of this article is to introduce the concept of total quasi- $\varphi$-asymptotically nonexpansive mapping which contains many kinds of mappings as its special cases and we prove a strong convergence theorem by using a hybrid method for finding a common element of the set of solutions for a generalized mixed equilibrium problems, the set of fixed points of a family of total quasi- $\varphi$ asymptotically nonexpansive mappings in uniformly smooth and strictly convex Banach space with the Kadec-Klee property. The results presented in the paper improve and extend some recent results.

\section{Preliminaries}

Throughout this article, we assume that all the Banach spaces are real. We denote by $\mathbb{N}$ and $\mathbb{R}$ the sets of positive integers and real numbers, respectively. Let $E$ be a Banach space and let $E^{*}$ be the topological dual of $E$. For all $x \in E$ and $x^{*} \in E^{*}$, we denote by 
$\left\langle x, x^{*}\right\rangle$ the value of $x^{*}$ at $x$. The mapping $J: E \rightarrow 2^{E^{*}}$ defined by

$$
J(x)=\left\{x^{*} \in E^{*}:\left\langle x, x^{*}\right\rangle=\|x\|^{2}=\left\|x^{*}\right\|^{2}\right\}, x \in E,
$$

is normalized duality mapping. We denote the weak convergence and the strong convergence of a sequence $\left\{x_{n}\right\}$ to $x$ by $x_{n} \rightarrow x$ and $x_{n} \rightarrow x$, respectively.

A Banach spaces $E$ is said to be strictly convex if $\frac{\|x+y\|}{2}<1$ for $x, y \in S(E)=\{z \in E$ : $\|$ $z \|=1\}$ with $x \neq y$. It is said to be uniformly convex if for any given $\varepsilon \in(0,2]$, there exists $\delta>0$ such that $\frac{\|x+y\|}{2}<1-\delta$ for $x, y \in S(E)$ with $\|x-y\| \geq \varepsilon$. E is said to have the Kadec-Klee property, if for any sequence $\left\{x_{n}\right\} \subset E$ such that $x_{n} \rightarrow x \in E$ and $\left\|x_{n}\right\|$ $\rightarrow\|x\|$, then $x_{n} \rightarrow x$.

Define $f: S(E) \times S(E) \times \mathbb{R} \backslash\{0\} \rightarrow \mathbb{R}$ by

$$
f(x, y, t)=\frac{\|x+t y\|-\|x\|}{t}
$$

for $x, y \in S(E)$ and $t \in \mathbb{R} \backslash\{0\}$. A norm of $E$ is said to be Gâteaux differentiable if $\lim _{t \rightarrow 0} f(x, y, t)$ has a limit for each $x, y \in S(E)$. In this case, $E$ is said to be smooth. We know that if $E$ is smooth, strictly convex, and reflexive, then the duality mapping $J$ is single valued, one to one, and onto. In this case, the inverse mapping $J^{-1}$ coincides with the duality mapping $J^{*}$ on $E^{*}$. See [14] for more details.

Remark 2.1 If $E$ is a reflexive and strictly convex Banach space, then $J^{1}$ is hemi-continuous, i.e., $J^{1}$ is norm-weak-continuous.

Let $\left\{C_{n}\right\}$ be a sequence of nonempty closed convex subset of a reflexive Banach space $E$. We define two subsets $s-L i_{n} C_{n}$ and $w-L s_{n} C_{n}$ as follows: $x \in s-L i_{n} C_{n}$ if and only if there exists $\left\{x_{n}\right\} \subset E$ such that $\left\{x_{n}\right\}$ converges strongly to $x$ and that $x_{n} \in C_{n}$ for all $n$ $\in \mathbb{N}$. Similarly, $y \in w-L s_{n} C_{n}$ if and only if there exists a subsequence $\left\{C_{n_{i}}\right\}$ of $\left\{C_{n}\right\}$ and a sequence $\left\{y_{i}\right\} \subset E$ such that $\left\{y_{i}\right\}$ converges weakly to $y$ and $y_{i} \in C_{n_{i}}$ for all $i \in \mathbb{N}$. We define the Mosco convergence [15] of $\left\{C_{n}\right\}$ as follows: If $C_{0}=s-L i_{n} C_{n}=w-$ $L s_{n} C_{n}$, then $\left\{C_{n}\right\}$ is said to be convergent to $C_{0}$ in the sense of Mosco and we write $C_{0}$ $=M-\lim _{n \rightarrow \infty} C_{n}$.

For more details, see [16].

Let $C$ be a nonempty closed convex subset of a smooth, strictly convex, and reflexive Banach space $E$. Then, for arbitrarily fixed $x \in E$, the function $y \propto\|x-y\|^{2}: C \rightarrow R^{+}$ has a unique minimizer $y_{x} \in C$. Using such a point, we define the metric projection $P_{C}$ by $P_{C} x=y_{x}=\arg \min _{y \in C}\|x-y\|^{2}$ for every $x \in E$. In a similar fashion, we can see that the function $y \propto \varphi(x, y): C \rightarrow R^{+}$has a unique minimizer $z_{x} \in C$. The generalized projection $\Pi_{C}$ of $E$ onto $C$ is defined by $\Pi_{C}=z_{x}=\arg \min _{y \in C} \varphi(x, y)$ for every $x \in E$; see [17].

The generalized projection $\Pi_{C}$ from $E$ onto $C$ is well defined, single valued and satisfies

$$
(\|x\|-\|y\|)^{2} \leq \phi(y, x) \leq(\|x\|+\|y\|)^{2}, \forall x, y \in E
$$

If $E$ is a Hilbert space, then $\varphi(y, x)=\|y-x\|^{2}$ and $\Pi_{C}$ is the metric projection $P_{C}$ of $E$ onto $C$.

It is well-known that the following conclusions hold: 
Lemma 2.1 [17,18]. Let $C$ be a nonempty closed convex subsets of a smooth, strictly convex, and reflexive Banach spaces. Then

$$
\phi\left(x, \Pi_{C} \gamma\right)+\phi\left(\Pi_{C} y, y\right) \leq \phi(x, y), \forall x \in C, y \in E .
$$

Lemma 2.2. Let $C$ be a nonempty closed convex subsets of a smooth, strictly convex, and reflexive Banach spaces $E$, let $x \in E$ and $z \in C$. Then the following conclusions hold:

(a) $z=\prod_{C} x \Leftrightarrow\langle y-z, J x-j z\rangle \leq 0, \forall y \in C$.

(b) For $x, y \in E, \varphi(x, y)=0$ if and only if $x=y$.

The following theorem proved by Tsukada [19] plays an important role in our results.

Theorem 2.1. Let $E$ be a smooth, reflexive, and strictly convex Banach spaces having the Kadec-Klee property. Let $\left\{K_{n}\right\}$ be a sequence of nonempty closed convex subsets of $E$. If $K_{0}=M-\lim _{n \rightarrow \infty} K_{n}$ exists and is nonempty, then $\left\{P_{K_{n}} x\right\}$ converges strongly to $P_{K_{0}} x$ for each $x \in C$.

Theorem 2.1 is still valid if we replace the metric projections with the generalized pro-jections as follows:

Theorem 2.2 Let $E$ be a smooth, reflexive, and strictly convex Banach spaces having the Kadec-Klee property. Let $\left\{K_{n}\right\}$ be a sequence of nonempty closed convex subsets of $E$. If $K_{0}=M-\lim _{n \rightarrow \infty} K_{n}$ exists and is nonempty, then $\left\{\prod_{K_{n}} x\right\}$ converges strongly to $\prod_{K_{0}} x$ for each $x \in C$.

For solving the equilibrium problem for bifunction $F: C \times C \rightarrow \mathbb{R}$, let us assume that $F$ satisfies the following conditions:

$\left(A_{1}\right) F(x, x)=0$ for all $x \in C$

$\left(A_{2}\right) F$ is monotone, i.e., $F(x, y)+F(y, x) \leq 0$ for all $x, y \in C$;

$\left(A_{3}\right)$ for each $x, y, z \in C$,

$$
\limsup _{t \downarrow 0} F(t z+(1-t) x, y) \leq F(x, y)
$$

$\left(A_{4}\right)$ for each $x \in C, y \propto F(x, y)$ is a convex and lower semicontinuous.

If an equilibrium bifunction $F: C \times C \rightarrow R$ satisfies conditions $\left(A_{1}\right)-\left(A_{4}\right)$, then we have the following results.

Lemma 2.3 [20]. Let $C$ be a nonempty closed convex subset of a smooth, strictly convex, and reflexive Banach spaces $E$, let $F$ be an equilibrium bifunction from $C \times C$ to $\mathbb{R}$ satisfying conditions $\left(A_{1}\right)-\left(A_{4}\right)$, let $r>0$ and let $x \in E$. Then, there exists $z \in C$ such that

$$
F(z, y)+\frac{1}{r}\langle y-z, J z-J x\rangle \geq 0, \forall y \in C
$$

Lemma 2.4 [21]. Let $C$ be a nonempty closed convex subset of a smooth, strictly convex, and reflexive Banach spaces $E$, let $F: C \times C \rightarrow \mathbb{R}$ be an equilibrium bifunction satisfying conditions $\left(A_{1}\right)-\left(A_{4}\right)$. For $r>0$ and $x \in E$, define a mapping $T_{r}: E \rightarrow C$ as follows:

$$
T_{r}(x)=\left\{z \in C: F(z, y)+\frac{1}{r}\langle y-z, J z-J x\rangle \geq 0, \forall y \in C\right\} .
$$


for all $x \in E$. Then, the following hold:

(1) $T_{r}$ is single-valued;

(2) $T_{r}$ is a firmly nonexpansive-type mapping, i.e., for any $x, y \in E$,

$$
\left\langle T_{r} x-T_{r} y, J T_{r} x-J T_{r} y\right\rangle \leq\left\langle T_{r} x-T_{r} y, J x-J y\right\rangle ;
$$

(3) $F\left(T_{r}\right)=\hat{F}\left(T_{r}\right)=E P(F)$;

(4) $E P(F)$ is a closed and convex set.

Lemma 2.5 [21]. Let $C$ be a nonempty closed convex subset of a smooth, strictly convex, and reflexive Banach spaces $E$, let $F: C \times C \rightarrow \mathbb{R}$ be an equilibrium bifunction satisfying conditions $\left(A_{1}\right)-\left(A_{4}\right)$. For $r>0$ and $x \in E$ and $q \in F\left(T_{r}\right)$,

$$
\phi\left(q, T_{r} x\right)+\phi\left(T_{r} x, x\right) \leq \phi(q, x)
$$

Lemma 2.6 [22]. Let $E$ be a uniformly convex Banach space and let $r>0$. Then there exists a strictly increasing, continuous, and convex function $g:[0,2 r] \rightarrow R$ such that $g$ (0) $=0$ and

$$
\|t x+(1-t) y\|^{2} \leq t\|x\|^{2}+(1-t)\|y\|^{2}-t(1-t) g(\|x-y\|) .
$$

\section{The main results}

Theorem 3.1. Let $E$ be a uniformly smooth and strictly convex Banach space with Kadec-Klee property and $C$ be a nonempty closed convex subset of $E$. $A: C \rightarrow E^{*}$ be a continuous and monotone mapping, $\psi: C \rightarrow \mathbb{R}$ be a lower semi-continuous and convex function and $F$ be a bifunction from $C \times C$ to $\mathbb{R}$ which satisfies the conditions $\left(A_{1}\right)-\left(A_{4}\right)$. Let $\left\{T_{\lambda}\right\}(\lambda \in \Lambda): C \rightarrow C$ be a family of uniformly $L_{\lambda}$-Lipschitzian continuous and uniformly total quasi- $\varphi$-asymptotically nonexpansive mappings such that $\mathfrak{F}=\bigcap_{\lambda \in \Lambda} F\left(T_{\lambda}\right) \bigcap G M E P \neq \emptyset$. Assume that $K=\sup \{\|u\|: u \in F\}<\infty$. Let $\left\{x_{n}\right\}$ be the sequence generated by $x_{1}=x \in C, C_{1}=C$ and

$$
\left\{\begin{array}{l}
y_{\lambda, n}=J^{-1}\left(\alpha_{n} J x_{n}+\left(1-\alpha_{n}\right) J T_{\lambda}^{n} x_{n}\right), \lambda \in \Lambda, \\
u_{\lambda, n} \in C \text { such that } \\
F\left(u_{\lambda, n}, y\right)+\left\langle A u_{\lambda, n}, y-u_{\lambda, n}\right\rangle+\psi(y)-\psi\left(u_{\lambda, n}\right) \\
\quad+\frac{1}{r_{\lambda, n}}\left\langle y-u_{\lambda, n}, J u_{\lambda, n}-J y_{\lambda, n}\right\rangle \geq 0, \forall y \in C, \lambda \in \Lambda \\
C_{n+1}=\left\{z \in C_{n}: \sup _{\lambda \in \Lambda} \phi\left(z, u_{\lambda, n}\right) \leq \phi\left(z, x_{n}\right)+\xi_{n}\right\}, \\
x_{n+1}=\Pi_{C_{n+1}} x, \forall n \geq 0 .
\end{array}\right.
$$

where $\xi_{n}=\left(1-\alpha_{n}\right)\left(v_{n} \sup _{u \in \mathrm{F}} \zeta\left(\varphi\left(u, x_{n}\right)\right)+\mu_{n}\right),\left\{\alpha_{n}\right\}$ is a sequence in [0,1] such that $\lim \inf _{n \rightarrow \infty} \alpha_{n}\left(1-\alpha_{n}\right)>0, \lim _{\inf _{n \rightarrow \infty}} \alpha_{n}<1$ and $\left\{r_{\lambda},{ }_{n}\right\}[a, \infty)$ for some $a>0$, then $\left\{x_{n}\right\}$ converge strongly to some point $x^{*}$ in $\mathrm{F}$.

Proof. We define a bifunction $G: C \times C \rightarrow R$ by

$$
G(z, y)=F(z, y)+\langle A z, y-z\rangle+\psi(y)-\psi(z), \forall z, y \in C .
$$

It is easy to prove that the bifunction $G$ satisfies conditions $\left(A_{1}\right)-\left(A_{4}\right)$.

Therefore, the generalized mixed equilibrium problem (1.1) is equivalent to the following equilibrium problem: find $z \in C$ such that

$$
G(z, y) \geq 0, \forall y \in C,
$$


and $G M E P=E P(G), F=G M E P \cap \cap_{\lambda \in \in \Lambda}=E P(G) \cap \cap_{\lambda \in \Lambda} F\left(T_{\lambda}\right)$. So, (3.1) can be written as:

$$
\left\{\begin{array}{l}
y_{\lambda, n}=J^{-1}\left(\alpha_{n} J x_{n}+\left(1-\alpha_{n}\right) J T_{\lambda}^{n} x_{n}\right) \text { for all } \lambda \in \Lambda, \\
u_{\lambda, n} \in C \text { such that } G\left(u_{\lambda, n}, y\right)+\frac{1}{r_{\lambda, n}}\left\langle y-u_{\lambda, n}, J u_{\lambda, n}-J y_{\lambda, n}\right\rangle \geq 0, \forall y \in C, \lambda \in \Lambda \\
C_{n+1}=\left\{z \in C_{n}: \sup _{\lambda \in \Lambda} \phi\left(z, u_{\lambda, n}\right) \leq \phi\left(z, x_{n}\right)+\xi_{n}\right\} \\
x_{n+1}=\Pi_{C_{n+1}} x, \forall n \geq 0 .
\end{array}\right.
$$

Since the bifunction $G$ satisfies conditions $\left(A_{1}\right)-\left(A_{4}\right)$, from Lemma 2.4, for given $r>0$ and $x \in E$, the mapping $W_{r}: E \rightarrow C$ defined by

$$
W_{r}(x)=\left\{z \in C: G(z, y)+\frac{1}{r}\langle y-z, J z-J x\rangle \geq 0, \forall y \in C\right\} .
$$

has the same properties as in Lemma 2.4.

Putting $u_{\lambda, n}=W_{r_{\lambda, n}} y_{\lambda, n}$ for all $n \in \mathbb{N}$, we have from Lemmas 2.4 and 2.5 that $W_{r_{\lambda, n}}$ is relatively nonexpansive.

We divide the proof of Theorem 3.1 into five steps:

Step 1. We first show that $C_{n}$ is closed and convex for every $n \in \mathbb{N}$.

From the definition of $\varphi$, we may show that

$$
\begin{aligned}
C_{n+1} & =\left\{z \in C_{n}: \sup _{\lambda \in \Lambda} \phi\left(z, u_{\lambda, n}\right) \leq \phi\left(z, x_{n}\right)+\xi_{n}\right\} \\
& =\cap_{\lambda \in \Lambda}\left\{z \in C_{n}: \phi\left(z, u_{\lambda, n}\right) \leq \phi\left(z, x_{n}\right)+\xi_{n}\right\} \\
& =\cap_{\lambda \in \Lambda}\left\{z \in C: 2\left\langle z, J x_{n}-J u_{\lambda, n}\right\rangle+\left\|u_{\lambda, n}\right\|^{2}-\left\|x_{n}\right\|^{2}-\xi_{n} \leq 0\right\} \cap C_{n},
\end{aligned}
$$

and thus $C_{n}$ is closed and convex for every $n \in \mathbb{N}$.

Step 2. Sequence $\left\{x_{n}\right\}$ is bounded.

In fact, since $x_{n}=\Pi_{C_{n}} x$, for any $p \in F$, from Lemma 2.1, we have

$$
\phi\left(x_{n}, x\right)=\phi\left(\Pi_{C_{n}} x, x\right) \leq \phi(p, x)-\phi\left(p, x_{n}\right) \leq \phi(p, x)
$$

This implies that the sequence $\left\{\varphi\left(x_{n}, x\right)\right\}$ is bounded, and so $\left\{x_{n}\right\}$ is bounded.

Step 3. Next we show that $\mathfrak{F} \subset C_{n}$ for each $n \in \mathbb{N}$.

For any $u \in \mathfrak{F}$, since $W_{r_{\lambda, n}}$ is relatively nonexpansive, $\left\{T_{\lambda}\right\}, \lambda \in \Lambda$ is uniformly total quasi- $\varphi$-asymptotically nonexpansive and $E^{*}$ is uniformly convex, it follows from Lemma 2.6 that

$$
\begin{aligned}
\phi\left(u, u_{\lambda, n}\right) & =\phi\left(u, W_{r_{\lambda, n}} y_{\lambda, n}\right) \leq \phi\left(u, y_{\lambda, n}\right) \\
& =\phi\left(u, J^{-1}\left(\alpha_{n} J x_{n}+\left(1-\alpha_{n}\right) J T_{\lambda}^{n} x_{n}\right)\right. \\
& =\|u\|^{2}-2\left\langle u, \alpha_{n} J x_{n}+\left(1-\alpha_{n}\right) J T_{\lambda}^{n} x_{n}\right\rangle+\left\|\alpha_{n} J x_{n}+\left(1-\alpha_{n}\right) J T_{\lambda}^{n} x_{n}\right\|^{2} \\
& \leq\|u\|^{2}-2 \alpha_{n}\left\langle u, J x_{n}\right\rangle-2\left(1-\alpha_{n}\right)\left\langle u, J T_{\lambda}^{n} x_{n}\right\rangle+\alpha_{n}\left\|J x_{n}\right\|^{2}+\left(1-\alpha_{n}\right)\left\|J T_{\lambda}^{n} x_{n}\right\|^{2} \\
& -\alpha_{n}\left(1-\alpha_{n}\right) g\left(\left\|J x_{n}-J T_{\lambda}^{n} x_{n}\right\|\right) \\
& =\alpha_{n} \phi\left(u, x_{n}\right)+\left(1-\alpha_{n}\right) \phi\left(u, T_{\lambda}^{n} x_{n}\right)-\alpha_{n}\left(1-\alpha_{n}\right) g\left(\left\|J x_{n}-J T_{\lambda}^{n} x_{n}\right\|\right) \\
& \leq \alpha_{n} \phi\left(u, x_{n}\right)+\left(1-\alpha_{n}\right)\left[\phi\left(u, x_{n}\right)+v_{n} \zeta\left(\phi\left(u, x_{n}\right)\right)+\mu_{n}\right]-\alpha_{n}\left(1-\alpha_{n}\right) g\left(\left\|J x_{n}-J T_{\lambda}^{n} x_{n}\right\|\right) \\
& =\phi\left(u, x_{n}\right)+\left(1-\alpha_{n}\right)\left[v_{n} \zeta\left(\phi\left(u, x_{n}\right)\right)+\mu_{n}\right]-\alpha_{n}\left(1-\alpha_{n}\right) g\left(\left\|J x_{n}-J T_{\lambda}^{n} x_{n}\right\|\right) \\
& \leq \phi\left(u, x_{n}\right)+\xi_{n}-\alpha_{n}\left(1-\alpha_{n}\right) g\left(\left\|J x_{n}-J T_{\lambda}^{n} x_{n}\right\|\right) \\
& \leq \phi\left(u, x_{n}\right)+\xi_{n} .
\end{aligned}
$$

This shows that $\left\{y_{\lambda, n}\right\}$ is bounded and $\sup _{\lambda \in \Lambda} \varphi\left(u, u_{\lambda, n}\right) \leq \varphi\left(u, x_{n}\right)+\xi_{n}$, i.e., $u \in$ $C_{n}$, this implies that 


$$
\mathfrak{F} \subset C_{n}, \forall n \in \mathbb{N} .
$$

Step 4. Now we prove that the $\operatorname{limit}_{\lim _{n \rightarrow \infty}} x_{n}$ exists.

Since $\mathfrak{F}$ is nonempty, $C_{n}$ is a nonempty closed convex subset of $E$ and thus $\Pi_{C_{n}}$ exists for every $n \in \mathbb{N}$. Hence $\left\{x_{n}\right\}$ is well defined. Also, since $\left\{C_{n}\right\}$ is a decreasing sequence of closed convex subsets of $C$ such that $C_{0}=\bigcap_{n=1}^{\infty} C_{n}$ is nonempty. Therefore, we have

$$
M-\lim _{n \rightarrow \infty} C_{n}=C_{0}=\bigcap_{n=1}^{\infty} C_{n} \neq \emptyset .
$$

By Theorem 2.2, $\left\{x_{n}\right\}=\left\{\Pi_{C_{n}} x\right\}$ converges strongly to $x^{*}=\Pi_{C_{0}} x$. Therefore, we have

$$
\left\|x_{n+1}-x_{n}\right\| \rightarrow 0 \text {. }
$$

Step 5. Next we prove $x^{*} \in \mathfrak{F}$.

(a) First, we prove $x^{*} \in \cap_{\lambda} \in \Lambda F\left(T_{\lambda}\right)$.

In fact, since $x_{n} \rightarrow x^{*}$, we have

$$
\phi\left(x_{n+1}, x_{n}\right) \rightarrow 0 \text {. }
$$

In view of $x_{n+1} \in C_{n+1}$, from the definition of $C_{n+1}$, we have

$$
\sup _{\lambda \in \Lambda} \phi\left(x_{n+1}, u_{\lambda, n}\right) \leq \phi\left(x_{n+1}, x_{n}\right)+\xi_{n} .
$$

From (3.5) and $\xi_{n} \rightarrow 0$, we have

$$
\sup _{\lambda \in \Lambda} \phi\left(x_{n+1}, u_{\lambda, n}\right) \rightarrow 0
$$

From (2.2) it yields $\sup _{\lambda \in \Lambda}\left(|| x_{n+1}\|-\| u_{\lambda, n} \|\right)^{2} \rightarrow 0$. Since $\left\|x_{n+1}\right\| \rightarrow\left\|x^{*}\right\|$, we have

$$
\left\|u_{\lambda, n}\right\| \rightarrow\left\|x^{*}\right\|(n \rightarrow \infty), \forall \lambda \in \Lambda
$$

Hence we have

$$
\left\|J u_{\lambda}, n\right\| \rightarrow\left\|J x^{*}\right\|(n \rightarrow \infty), \forall \lambda \in \Lambda
$$

This implies that $\left\{J u_{\lambda}, n\right\}$ is uniformly bounded in $E^{*}$. Since $E$ is reflexive, and so is $E^{*}$. We can assume that $J u_{\lambda, n} \rightarrow f_{0} \in E^{*}$. In view of the reflexive of $E$, we see that $J(E)=$ $E^{*}$. Hence there exists $p \in E$ such that $J p=f_{0}$. Since

$$
\begin{aligned}
\phi\left(x_{n+1}, u_{\lambda, n}\right) & =\left\|x_{n+1}\right\|^{2}-2\left\langle x_{n+1}, J u_{\lambda, n}\right\rangle+\left\|u_{\lambda, n}\right\|^{2} \\
& =\left\|x_{n+1}\right\|^{2}-2\left\langle x_{n+1}, J u_{\lambda, n}\right\rangle+\left\|u_{\lambda, n}\right\|^{2}
\end{aligned}
$$

Taking $\lim \inf _{n \rightarrow \infty}$ on the both sides of equality above and in view of the weak lower semicontinuity of norm $\|\cdot\|$, it yields that

$$
\begin{aligned}
0 & \geq\left\|x^{*}\right\|^{2}-2\left\langle x^{*}, f_{0}\right\rangle+\left\|f_{0}\right\|^{2}=\left\|x^{*}\right\|^{2}-2\left\langle x^{*}, J p\right\rangle+\|J p\|^{2} \\
& =\left\|x^{*}\right\|^{2}-2\left\langle x^{*}, J p\right\rangle+\|p\|^{2}=\phi\left(x^{*}, p\right)
\end{aligned}
$$

i.e., $x^{*}=p$. This implies that $f_{0}=J x^{*}$, and so $J u_{\lambda, n} \rightarrow J x^{*}, \forall \lambda \in \Lambda$. It follows from (3.7) and the Kadec-Klee property of $E^{*}$ that $J u_{\lambda, n} \rightarrow J x^{*}(n \rightarrow \infty)$. Note that $J^{-1}: E^{*} \rightarrow$ $E$ is hemi-continuous, it yields that $u_{\lambda, n} \rightarrow x^{*}$. In view of (3.6) and the Kadec-Klee 
property of $E$, we have

$$
\lim _{n \rightarrow \infty} u_{\lambda, n}=x^{*} . \forall \lambda \in \Lambda
$$

From (3.8), we have

$$
\lim _{n \rightarrow \infty}\left\|x_{n}-u_{\lambda, n}\right\|=0, \quad \forall \lambda \in \Lambda
$$

Since $J$ is uniformly continuous, we have that

$$
\left\|J x_{n}-J u_{\lambda, n}\right\| \rightarrow 0, \forall \lambda \in \Lambda
$$

From (3.9) and (3.10), we have

$$
\begin{aligned}
\phi\left(u, x_{n}\right)-\phi\left(u, u_{\lambda, n}\right) & =\left\|x_{n}\right\|^{2}-\left\|u_{\lambda, n}\right\|^{2}-2\left\langle u, J x_{n}-J u_{\lambda, n}\right\rangle \\
& \leq\left\|\left|\left\|x_{n}\right\|^{2}-\left\|u_{\lambda, n}\right\|^{2}\right|+2\left|\left\langle u, J x_{n}-J u_{\lambda, n}\right\rangle\right|\right. \\
& \leq\left|\left\|x_{n}\right\|-\left\|u_{\lambda, n}\right\|\right|\left(\left\|x_{n}\right\|+\left\|u_{\lambda, n}\right\|\right)+2\|u\| \cdot\left\|J x_{n}-J u_{\lambda, n}\right\| \\
& \leq\left\|x_{n}-u_{\lambda, n}\right\|\left(\left\|x_{n}\right\|+\left\|u_{\lambda, n}\right\|\right)+2\|u\| \cdot\left\|J x_{n}-J u_{\lambda, n}\right\| . \\
& \rightarrow 0
\end{aligned}
$$

It follows from (3.3), (3.11) and $\xi_{n} \rightarrow 0$ that

$$
\alpha_{n}\left(1-\alpha_{n}\right) g\left(|| J x_{n}-J T_{\lambda}^{n} x_{n}||\right) \leq \phi\left(u, x_{n}\right)-\phi\left(u, u_{\lambda, n}+\xi_{n} \rightarrow 0 .\right.
$$

In view of condition $\lim \inf _{n \rightarrow \infty} \alpha_{n}\left(1-\alpha_{n}\right)>0$, we see that

$$
g\left(|| J x_{n}-J T_{\lambda}^{n} x_{n} \|\right) \rightarrow 0(\text { as } n \rightarrow \infty)
$$

It follows from the property of $g$ that

$$
\left\|J x_{n}-J T_{\lambda}^{n} x_{n}\right\| \rightarrow 0(\text { as } n \rightarrow \infty) .
$$

Since $x_{n} \rightarrow x^{*}$ and so $J x_{n} \rightarrow J x^{*}$. From (3.13) we have

$$
J T_{\lambda}^{n} x_{n} \rightarrow J x^{*}(\text { as } n \rightarrow \infty) .
$$

Since $J^{-1}: E^{*} \rightarrow E$ is hemi-continuous, it follows that

$$
T_{\lambda}^{n} x_{n} \rightarrow x^{*}, \forall \lambda \in \Lambda
$$

On the other hand, for each $\lambda \in \Lambda$ we have

$$
\left|\left\|T_{\lambda}^{n} x_{n}\right\|-\left\|x^{*}\right\|\right|=\left|\left\|J\left(T_{\lambda}^{n} x_{n}\right)\right\|-\left\|J x^{*}\right\|\right| \leq\left\|J\left(T_{\lambda}^{n} x_{n}\right)-J x^{*}\right\| \rightarrow 0 \quad(\text { as } n \rightarrow \infty) .
$$

This together with (3.14) shows that

$$
T_{\lambda}^{n} x_{n} \rightarrow x^{*}, \forall \lambda \in \Lambda
$$

Furthermore, by the assumption that for each $\lambda \in \Lambda, T_{\lambda}$ is uniformly $L_{\lambda}$-Lipschitz continuous, hence from (3.4) and (3.15), we have

$$
\begin{aligned}
\left\|T_{\lambda}^{n+1} x_{n}-T_{\lambda}^{n} x_{n}\right\| \leq & \left\|T_{\lambda}^{n+1} x_{n}-T_{\lambda}^{n+1} x_{n+1}\right\|+\left\|T_{\lambda}^{n+1} x_{n+1}-x_{n+1}\right\| \\
& +\left\|x_{n+1}-x_{n}\right\|+\left\|x_{n}-T_{\lambda}^{n} x_{n}\right\| \\
\leq & \left(L_{\lambda}+1\right)\left\|x_{n+1}-x_{n}\right\|+\left\|T_{\lambda}^{n+1} x_{n+1}-x_{n+1}\right\|+\left\|x_{n}-T_{\lambda}^{n} x_{n}\right\| \\
\rightarrow & 0
\end{aligned}
$$


This implies that $T_{\lambda}^{n+1} x_{n} \rightarrow x^{*}$, i.e., $T_{\lambda} T_{\lambda}^{n} x_{n} \rightarrow x^{*}$. In view of (3.15) and $T_{\lambda}$ is uniformly Lipschitzian continuous, it yields that $T_{\lambda} x^{*}=x^{*}, \forall \lambda \in \Lambda$. This implies that $x^{*} \in \cap_{\lambda \in \Lambda} F\left(T_{\lambda}\right)$.

(b) Next, we prove $x^{*} \in E P(G)$.

Since

$$
\begin{aligned}
\phi\left(u_{\lambda, n}, y_{\lambda, n}\right) & =\phi\left(W_{r_{\lambda, n}} y_{\lambda, n}, y_{\lambda, n}\right) \\
& \leq \phi\left(u, y_{\lambda, n}\right)-\phi\left(u, W_{r_{\lambda, n}} y_{\lambda, n}\right) \\
& \leq \phi\left(u, x_{n}\right)+\xi_{n}-\phi\left(u, W_{r_{\lambda, n}} y_{\lambda, n}\right) \\
& =\phi\left(u, x_{n}\right)+\xi_{n}-\phi\left(u, u_{\lambda, n}\right) .
\end{aligned}
$$

Hence it follows from (3.11) and (3.16) that

$$
\lim _{n \rightarrow \infty} \phi\left(u_{\lambda, n}, y_{\lambda, n}\right)=0
$$

From (2.2) and (3.17) it yields $\left(\left\|u_{\lambda, n}\right\|-\left\|y_{\lambda, n}\right\|\right)^{2} \rightarrow 0$. Since $\left\|u_{\lambda, n}\right\| \rightarrow\left\|x^{* *}\right\|$, we have

$$
\left\|y_{\lambda, n}\right\| \rightarrow\left\|x^{*}\right\|(n \rightarrow \infty) .
$$

Hence we have

$$
\left\|J y_{\lambda, n}\right\| \rightarrow\left\|J x^{*}\right\|(n \rightarrow \infty)
$$

This implies that $\left\{y y_{\lambda, n}\right\}$ is bounded in $E^{*}$. Since $E$ is reflexive, and so is $E^{*}$. we can assume that $J y_{\lambda, n} \rightarrow g_{0} \in E^{*}$. In view of the reflexive of $E$, we see that $J(E)=E^{*}$. Hence there exists $y \in E$ such that $J y=g_{0}$. Since

$$
\begin{aligned}
\phi\left(u_{\lambda, n}, y_{\lambda, n}\right) & =\left\|u_{\lambda, n}\right\|^{2}-2\left\langle u_{\lambda, n}, J y_{\lambda, n}\right\rangle+\left\|y_{\lambda, n}\right\|^{2} \\
& =\left\|u_{\lambda, n}\right\|^{2}-2\left\langle u_{\lambda, n}, J y_{\lambda, n}\right\rangle+\left\|J y_{\lambda, n}\right\|^{2}
\end{aligned}
$$

Taking $\lim \inf _{n \rightarrow \infty}$ on the both sides of equality above and in view of the weak lower semicontinuity of norm $\|\cdot\|$, it yields that

$$
\begin{aligned}
0 & \geq\left\|x^{*}\right\|^{2}-2\left\langle x^{*}, g_{0}\right\rangle+\left\|g_{0}\right\|^{2}=\left\|x^{*}\right\|^{2}-2\left\langle x^{*}, J y\right\rangle+\|J y\|^{2} \\
& =\left\|x^{*}\right\|^{2}-2\left\langle x^{*}, J y\right\rangle+\|y\|^{2}=\phi\left(x^{*}, y\right)
\end{aligned}
$$

i.e., $x^{*}=y$. This implies that $g_{0}=J x^{*}$, and so $J y_{\lambda, n} \rightarrow J x^{*}$. It follows from (3.19) and the Kadec-Klee property of $E^{*}$ that $J y_{n}(\lambda) \rightarrow J x^{*}(n \rightarrow \infty)$. Note that $J^{1}: E^{*} \rightarrow E$ is hemicontinuous, it yields that $y_{\lambda, n} \rightarrow x^{*}$. It follows from (3.18) and the Kadec-Klee property of $E$ that

$$
\lim _{n \rightarrow \infty} y_{\lambda, n}=x^{*} .
$$

Since $u_{\lambda, n} \rightarrow x^{*}$, from (3.20), we have

$$
\lim _{n \rightarrow \infty}\left\|u_{\lambda, n}-y_{\lambda, n}\right\|=0 .
$$

Since $J$ is uniformly norm-to-norm continuous on bounded sets, from (3.21), we have

$$
\lim _{n \rightarrow \infty} \mid\left\|J u_{\lambda, n}-J y_{\lambda, n}\right\|=0 .
$$


From $r_{\lambda, n} \geq a$, we have

$$
\lim _{n \rightarrow \infty} \frac{\left\|J u_{\lambda, n}-J y_{\lambda, n}\right\|}{r_{\lambda, n}}=0
$$

By $u_{\lambda, n}=W_{r_{\lambda, n}} y_{\lambda, n}$, we have

$$
G\left(u_{\lambda, n}, y\right)+\frac{1}{r_{\lambda, n}}\left\langle y-u_{\lambda, n}, J u_{\lambda, n}-J y_{\lambda, n}\right\rangle \geq 0, \quad \forall y \in C
$$

From condition $\left(A_{2}\right)$, we have

$$
\frac{1}{r_{\lambda, n}}\left\langle y-u_{\lambda, n}, J u_{\lambda, n}-J y_{\lambda, n}\right\rangle \geq-G\left(u_{\lambda, n}, y\right) \geq G\left(y, u_{\lambda, n}\right), \quad \forall y \in C .
$$

Since $G(x, \cdot)$ is convex and lower semicontinuous, it is also weakly lower semicontinuous, letting $n \rightarrow \infty$, we have from (3.25) and $\left(A_{4}\right)$ that

$$
G\left(y, x^{*}\right) \leq 0, \forall y \in C .
$$

For any $t$ with $0<t \leq 1$ and $y \in C$, let $y_{t}=t y+(1-t) x^{*}$. Since $y \in C$ and hence $G$ $\left(y_{t}, x^{*}\right) \leq 0$, from conditions $\left(A_{1}\right)$ and $\left(A_{4}\right)$, we have

$$
0=G\left(y_{t}, y_{t}\right) \leq t G\left(y_{t}, y\right)+(1-t) G\left(y_{t}, x^{*}\right) \leq t G\left(y_{t}, y\right)
$$

This implies that $G\left(y_{t}, y\right) \geq 0$. Hence from condition $\left(A_{3}\right)$, we have $G\left(x^{*}, y\right) \geq 0$ for all $y \in C$, and hence $x^{*} \in E P(G)$.

This completes the proof of Theorem 3.1.

The proof of Theorem 3.1 shows that the properties of generalized projections used in the iterative scheme do not interact with the properties of mappings $\left\{T_{\lambda}\right\}$.

Theorem 3.2. Let $E$ be a uniformly smooth and strictly convex Banach space with Kadec-Klee property and $C$ be a nonempty closed convex subset of $E$. $A: C \rightarrow E^{*}$ be a continuous and monotone mapping, $\psi: C \rightarrow \mathbb{R}$ be a lower semi-continuous and convex function and $F$ be a bifunction from $C \times C$ to $\mathbb{R}$ which satisfies the conditions $\left(A_{1}\right)-\left(A_{4}\right)$. Let $\left\{T_{\lambda}\right\}(\lambda \in \Lambda): C \rightarrow C$ be a family of uniformly $L_{\lambda}$-Lipschitzian continuous and uniformly quasi- $\varphi$-asymptotically nonexpansive mappings such that $\mathfrak{F}=\bigcap_{\lambda \in \Lambda} F\left(T_{\lambda}\right) \bigcap G M E P \neq \emptyset$. Assume that $K=\sup \{\|u\|: u \in \mathfrak{F}\}<\infty$. Let $\left\{x_{n}\right\}$ be the sequence generated by $x_{1}=x \in C, C_{1}=C$ and

$$
\left\{\begin{array}{l}
y_{\lambda, n}=J^{-1}\left(\alpha_{n} J x_{n}+\left(1-\alpha_{n}\right) J T_{\lambda}^{n} x_{n}\right), \lambda \in \Lambda, \\
u_{\lambda, n} \in C \text { such that } \\
F\left(u_{\lambda, n}, y\right)+\left\langle A u_{\lambda, n}, y-u_{\lambda, n}\right\rangle+\psi(y)-\psi\left(u_{\lambda, n}\right) \\
\quad+\frac{1}{r_{\lambda, n}}\left\langle y-u_{\lambda, n}, J u_{\lambda, n}-J y_{\lambda, n}\right\rangle \geq 0, \forall y \in C, \lambda \in \Lambda \\
C_{n+1}=\left\{z \in C_{n}: \sup _{\lambda \in \Lambda} \phi\left(z, u_{\lambda, n}\right) \leq \phi\left(z, x_{n}\right)+\xi_{n}\right\}, \\
x_{n+1}=\Pi_{C_{n+1}} x, \forall n \geq 0 .
\end{array}\right.
$$

where $\xi_{n}=\left(1-\alpha_{n}\right)\left(v_{n} \sup _{u \in \mathfrak{F}} \zeta\left(\phi\left(u, x_{n}\right)\right)+\mu_{n}\right),\left\{\alpha_{n}\right\}$ is a sequence in $0[1]$ such that $\lim \inf _{n \rightarrow \infty} \alpha_{n}\left(1-\alpha_{n}\right)>0, \lim _{\inf _{n \rightarrow \infty}} \alpha_{n}<1$ and $\left\{r_{\lambda, n}\right\} \subset[a, \infty)$ for some $a>0$, then $\left\{x_{n}\right\}$ converge strongly to some point $x^{*}$ in $F$. 
Proof. In Theorem 3.1 take $\zeta(t)=t, v_{n}=k_{n}-1, \mu_{n}=0$. Therefore the conclusion of Theorem 3.2 can be obtained form Theorem 3.1.

\section{Acknowledgements}

This study was supported by Scientific Research Fund of Sichuan Provincial Education Department (11ZB146)(2012), the natural Science Foundation of Yibin University (2011Z08) and Scientific Research Fund of Science Technology Department of Sichuan Province (2011JYZ011).

\section{Author details}

${ }^{1}$ Department of Mathematics, Yibin university, Yibin, Sichuan 644007, China ${ }^{2}$ Department of Mathematics, College of Statistics and Mathematics, Yunnan University of Finance and Economics, Kunming, Yunnan 650221, China

\section{Authors' contributions}

All the authors contributed equally to the writing of the present article. All authors read and approved the final manuscript.

\section{Competing interests}

The authors declare that they have no competing interests.

Received: 23 December 2011 Accepted: 1 May 2012 Published: 1 May 2012

\section{References}

1. Ceng, LC, Yao, JC: A hybrid iterative scheme for mixed equilibrium problems and fixed point problems. J Comput Appl Math. 214, 186-201 (2008)

2. Takahashi, S, Takahashi, W: Strong convergence theorem for a generalized equilibrium problem and a nonexpansive mapping in a Hilbert space. Nonlinear Anal. 69(3):1025-1033 (2008)

3. Tada, A, Takahashi, W: Strong convergence theorem for an equilibrium problem and a nonexpansive mapping. JOptim Theory Appl. 133, 359-370 (2007)

4. Takahashi, S, Takahashi, W: Viscosity approximation methods for equilibrium problem and fixed point problems in Hilbert spaces. J Math Anal Appl. 331, 506-515 (2007)

5. Kimura, Y, Takahashi, W: On a hybrid method for a family of relatively nonexpansive mappings in a Banach space. J Math Anal Appl. 357, 356-363 (2009)

6. Takahashi, W: Nonlinear Functional Analysis. Yokohama Publishers, Yokohama (2000)

7. Liu, M, Chang, SS, Zuo, P: On a hybrid method for generalized mixed equilibrium problem and fixed point problem of a family of quasi- $\varphi$-asymptotically nonexpansive mappings in Banach space. Fixed Point Theory and Applications 2010, 18 (2010). Article ID 157278

8. Liu, M, Chang, SS, Zuo, P. Shrinking projection method of common solutions for generalized equilibrium quasi- $\varphi$ nonexpansive mapping and relatively nonexpansive mapping. J Inequal Appl 2010, 15 (2010). Article ID 101690

9. Chang, SS: The generalized mixed equilibrium problem in Banach. Appl Math Mech. 30, 1105-1112 (2009)

10. Su, YF, Zhang, HK: Strong convergence theorems for two countable families of weak relatively nonexpansive mappings and applications. Nonlinear Anal. 73, 3890-3906 (2010)

11. Zegeye, $\mathrm{H}$, Shahzad, $\mathrm{N}$ : Strong convergence theorems for monotone mappings and relatively weak nonexpansive mappings. Nonlinear Anal Theory Methods Appl. 70(7):2707-2716 (2009)

12. Zhang, HC, Su, YF: Strong convergence of modified hybrid algorithm for quasi- $\varphi$-asymptotically nonexpansive mappings. Commun Korean Math Soc. 24(4):539-551 (2009)

13. Qin, XL, Cho, YS, Kang, SM: On hybrid projection methods for asymptotically quasi- $\varphi$-nonexpansive mappings. Appl Math Comput. 215(11):3874-3883 (2010)

14. Goebel, K, Reich, S: Uniform convexity, hyperbolic geometry, and nonexpansive mappings. In Monogr Textbooks Pure Appl Math, vol. 83,Marcel Dekker Inc., New York (1984)

15. Mosco, U: Convergence of convex sets and of solutions of variational inequalities. Adv Math. 3, $510-585$ (1969)

16. Beer, G: Topologies on Closed and Closed Convex Sets. Kluwer Academic Publishers Group, Dordrecht (1993)

17. Alber, Yl: Metric and generalized projection operators in Banach spaces: properties and applications. In: Kartsators AG (ed.) Theory and Applications of Nonlinear Operators of Accretive and Monotone Type, vol. 178, pp. 15-50. Dekker, New York, NY, USA (1996). of Lecture Notes in Pure and Applied Mathematics

18. Kamimura, S, Takahashi, W: Strong convergence of a proximal-type algorithm in a Banach space. SLAM J Optim. 13(3):938-945 (2002)

19. Tsukada, M: Convergence of best approximations in a smooth Banach space. J Approx Theory. 40, 301-309 (1984)

20. Blum, E, Oettli, W: From optimization and varitional inequalities to equilibrium problems. Math Student. 63, 123-145 (1994)

21. Takahashi, W, Zembayashi, K: Strong convergence theorems by a new hybrid method for equilibrium problems and relatively nonexpansive mappings. Fixed Point Theory and Applications 2008, 11 (2008). Article ID 528476. doi:10.1155/ 2008/528476

22. Xu, HK: Inequalities in Banach spaces with applications. Nonlinear Anal: Theory Methods Appl. 16(12):1127-1138 (1991)

doi:10.1186/1687-1812-2012-70

Cite this article as: Zuo et al:: On a hybrid algorithm for a family of total quasi- $\varphi$-asymptotically nonexpansive mappings in Banach spaces. Fixed Point Theory and Applications 2012 2012:70. 